\title{
LLLT IN THE SYMPTOMATIC TREATMENT OF ORAL LICHEN PLANUS
}

\author{
Applicant: Carlo Fornaini MD; DDS, MSc \\ Oral Medicine and Laser-Assisted Surgery Unit \\ Dental School, Faculty of Medicine, University of Parma, Italy
}

\section{Background and aims}

Oral Lichen Planus (OLP) is a chronic inflammatory disorder that normally can affect oral mucosa surfaces but that may also interest non-oral epithelial surfaces such as skin, genitalia, nails and scalp.

The posterior buccal mucosa is most frequently involved, followed by the tongue, gingiva and vermilion of the lower lip.

It affects mostly women between the fifth and the sixth decades of life but the disease may also affect individuals aged below 18 years: in this case, while the clinical features are the same as those that present in older adults, the prognosis is more benign.

OLP is often asymptomatic but in the case there are areas of erosion or ulceration the patient may have variable amounts of discomfort, particularly when eating spicy or acidic type foods.

Although OLP can spontaneously regress, many lesions eventually require treatment. The most commonly used agents for the treatment of OLP are topical corticosteroids.

Intralesional and systemic corticosteroids are also used. Topical and systemic medications include immunosuppressant, such as cyclosporine and tacrolimus and topic or systemic retinoids.

The treatment of refractory disease has also been approached with other agents, including thalidomide. The aim of this clinical work was to analyze the utilization of laser beam at low power (LLLT) to reduce the discomfort and inflammation due to the OLP.

Addressee for Correspondence:

Carlo Fornaini MD; DDS, MSc

Oral Medicine and Laser-Assisted Surgery Unit

Dental School, Faculty of Medicine

University of Parma, Italy

E-mail: carlo@fornainident.it

\section{Subjects and Methods}

The subjects treated in this clinical study were nineteen patients with presence of OLP lesions localized in the tongue. The subjects were all women and the age was from 45 to 84. (Fig. 1)

All these subjects referred a situation of great discomfort due to the difficulty to eat due to the increase of the oral symptomatology.

In every patient a biopsy of oral lesions was performed before starting the laser irradiation sessions, in order to confirm, also by a histological point of view, the clinical diagnosis.

A part of these patients (14) had done a pharmacological treatment, by topical assumption of cortisone and nistatine, without significant results. (Fig. 2)

For the irradiation in defocused mode, it was used a KTP laser (LaseMar 500, Eufoton, Trieste, Italy)

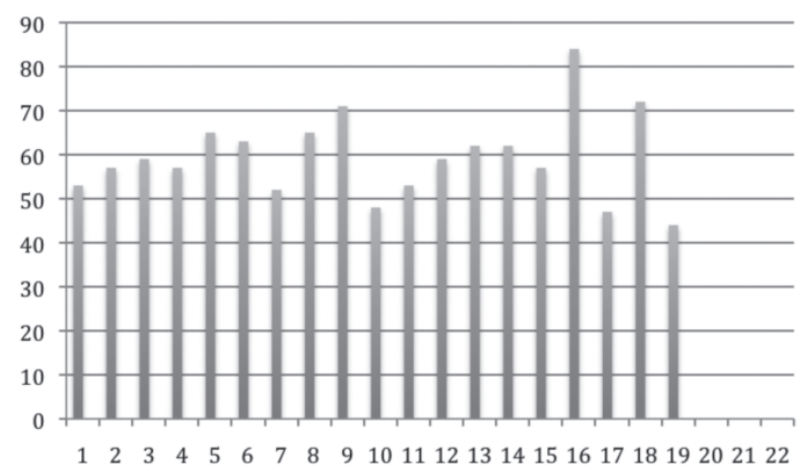

Fig. 1: Age distribution (Average 59.47)

Manuscript received: January 7th, 2012

Accepted for publication: February 20th, 2012

J-STAGE advance published: February 23rd, 2012 


\section{ILLIS CASE REPORT}

which emits a green visible beam (532 nm) with by $600 \mu \mathrm{m}$ fibrotic delivery system and a handpiece consisting of a $40 \mathrm{~mm}$ diameter defocusing lens.

The treatment was performed in 6 different sessions (twice weekly) of 4 minutes each, distributed in steps of 1 minute of irradiation with $30 \mathrm{sec}$. of pause

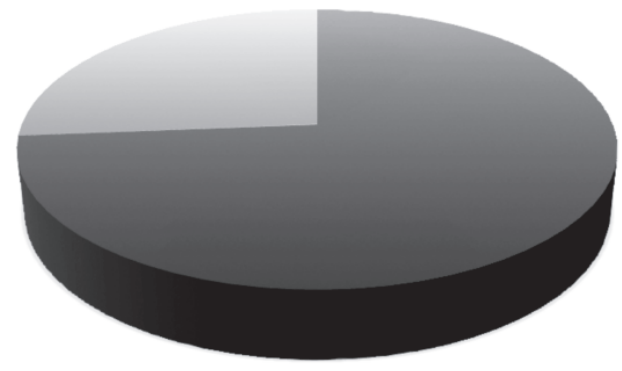

Fig. 2: Percentage of the patients previously pharmacologically treated $(73.68 \%)$

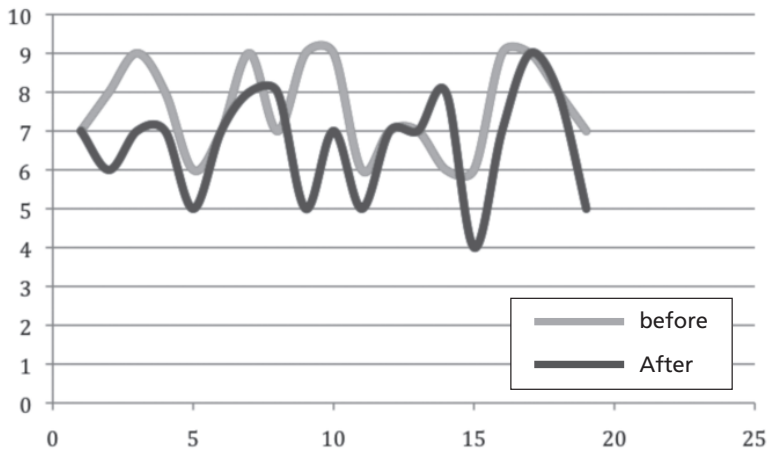

and the Fluence obtained, in continuous wave, was of $4 \mathrm{~J} / \mathrm{cm}^{2}$.

Before, just after the laser treatment and 1 month after, all the patients expressed the degree of their discomfort with NRS system, by giving a numerical score between 0 (absence of discomfort) and 10 (maximum

Fig. 4: Morphological evaluation before and after by the two clinicians.

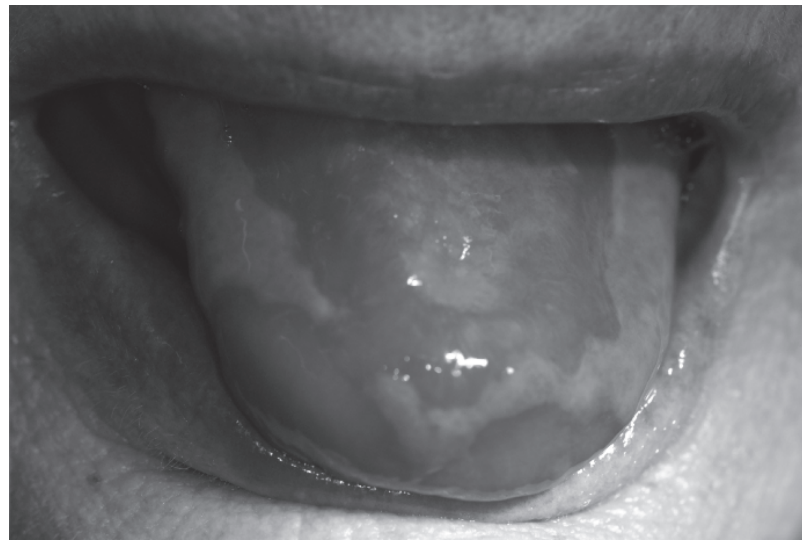

Fig. 5: The tongue lesions before laser treatment

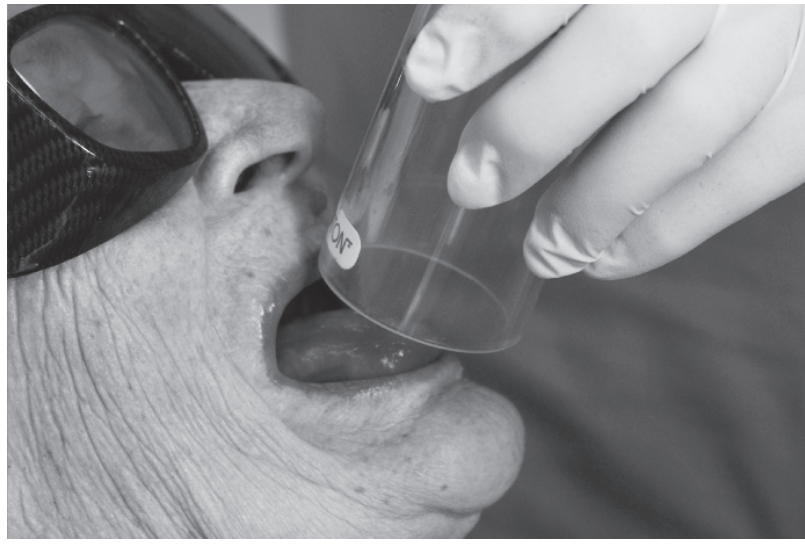

Fig. 6: Tongue LLLT irradiation 


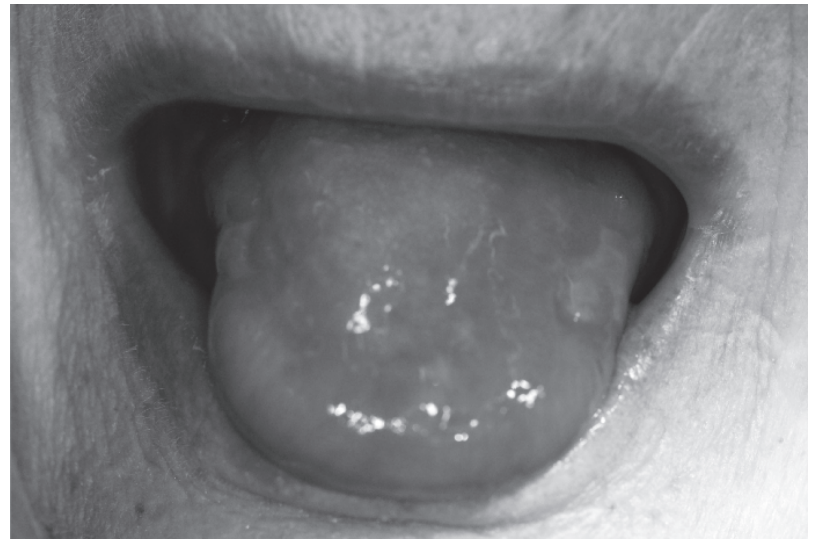

Fig. 7: Aspect of the tongue after the LLLT treatment

of discomfort). (Fig. 3)

Another evaluation, based on morphological aspects of the lesions and expressed by a score from 0 (absence of lesions) to 10 (most important lesions), was given also, before and after laser treatment, by two different clinicians. (Fig. 4)

\section{Case Report}

Patient VA, 84 female, came to our clinics with important symptomatic lesions present in the tongue since several years. (Fig. 5) By anamnesis examination, the patient resulted positive for debate and hypertension and she said to be in treatment also for a depression, which had an important increase with the aggravation of the oral disease, and the consequent impossibility to have a correct diet and to wear her removable prosthetics appliances.

The patient started a topic treatment consisting in mouthwashes with cortisone (Clobetasolo 0.05\%) and antifungal (Nistatina). One month after, because no relief of symptoms was noted, it was decided to start a LLLT treatment with the device and parameters before described. (Fig. 6)

After 3 sessions, the patients referred an important relief of the pain and discomfort and it was also noted a reduction of the lesions in the tongue. (Fig. 7) The follow-up after the treatment and one month after demonstrated a great reduction of the symptoms.

\section{Conclusion}

OLP is a chronic inflammatory oral mucosal disease in which cell mediated immunity plays a major role. In the nineteen patients treated by LLLT therapy it was noticed an important reduction of the symptomatology consisting in discomfort and pain with the consequent difficulty to eat. The follow-up demonstrated also a reduction in the number, dimension and gravity of the oral lesions.

Even if, due to the small number of the patients treated, this clinical work must be considered as a preliminary study, it demonstrated that LLLT may be used a to reduce the symptoms of oral localization of Lichen Planus, without side effects. 